University of Texas at El Paso

ScholarWorks@UTEP

4-2013

\title{
Towards a Better Understanding of Space-Time Causality: Kolmogorov Complexity and Causality as a Matter of Degree
}

Vladik Kreinovich

The University of Texas at El Paso, vladik@utep.edu

Andres Ortiz

The University of Texas at El Paso, aortiz19@miners.utep.edu

Follow this and additional works at: https://scholarworks.utep.edu/cs_techrep

Part of the Mathematics Commons, and the Physics Commons

Comments:

Technical Report: UTEP-CS-12-24c

To appear in Proceedings of the joint World Congress of the International Fuzzy Systems Association and Annual Conference of the North American Fuzzy Information Processing Society IFSA/NAFIPS'2013, Edmonton, Canada, June 24-28, 2013.

\section{Recommended Citation}

Kreinovich, Vladik and Ortiz, Andres, "Towards a Better Understanding of Space-Time Causality: Kolmogorov Complexity and Causality as a Matter of Degree" (2013). Departmental Technical Reports (CS). 718.

https://scholarworks.utep.edu/cs_techrep/718

This Article is brought to you for free and open access by the Computer Science at ScholarWorks@UTEP. It has been accepted for inclusion in Departmental Technical Reports (CS) by an authorized administrator of ScholarWorks@UTEP.For more information, please contact Iweber@utep.edu. 


\title{
Towards a Better Understanding of Space-Time Causality: Kolmogorov Complexity and Causality as a Matter of Degree
}

\author{
Vladik Kreinovich ${ }^{1}$ and Andres Ortiz ${ }^{2,3}$ \\ ${ }^{1}$ Department of Computer Science \\ ${ }^{2}$ Department of Mathematical Sciences \\ ${ }^{3}$ Department of Physics \\ University of Texas at El Paso \\ $500 \mathrm{~W}$. University \\ El Paso, TX 79968, USA \\ vladik@utep.edu, aortiz19@miners.utep.edu
}

\begin{abstract}
Space-time causality is one of the fundamental notions of modern physics; however, it is difficult to define in observational physical terms. Intuitively, the fact that a spacetime event $e=(t, x)$ can causally influence an event $e^{\prime}=\left(t^{\prime}, x^{\prime}\right)$ means that what we do in the vicinity of $e$ changes what we observe at $e^{\prime}$. If we had two copies of the Universe, we could perform some action at $e$ in one copy but not in another copy; if we then observe the difference at $e^{\prime}$, this would be an indication of causality. However, we only observe one Universe, in which we either perform the action or we do not. At first glance, it may seem that in this case, there is no meaningful way to provide an operational definition of causality. In this paper, we show that such a definition is possible if we use the notions of algorithmic randomness and Kolmogorov complexity. The resulting definition leads to a somewhat unexpected consequence: that space-time causality is a matter of degree.
\end{abstract}

\section{Defining Causality Is Important}

Space-time causality is important. Causal relation between space-time events (i.e., points in space-time) is one of the fundamental notions of physics; see, e.g., [1], [3]). Because of this, many fundamental physical theories describe, among other things, the causal relation between space-time events.

According to modern physics, space-time causal relation is non-trivial. In Newton's physics, it was assumed that influences can propagate with an arbitrary speed, constituting, in effect, immediate action-at-a-distance. Under this assumption, an event $e=(t, x)$ occurring at moment $t$ at location $x$ can influence an event $e^{\prime}=\left(t^{\prime}, x^{\prime}\right)$ occurring at moment $t^{\prime}$ at location $x^{\prime}$ if and only if the second event occurs later than the first one, i.e., if and only if $t<t^{\prime}$.

In special relativity, the speeds of all the processes are limited by the speed of light $c$. In this theory, an event $e=(t, x)$ can influence an event $e^{\prime}=\left(t^{\prime}, x^{\prime}\right)$ if during the time $t^{\prime}-t$, the faster possible process - light - can cover the distance $d\left(x, x^{\prime}\right)$ between locations $x$ and $x^{\prime}$, i.e., if

$$
c \cdot\left(t^{\prime}-t\right) \geq d\left(x, x^{\prime}\right) .
$$

In the general relativity theory, the space-time is curved, so the corresponding causal relation is even more complex. This relation is also complex in alternative gravitation theories; see, e.g., [3].

Need for experimental verification of space-time casuality. Different theories, in general, make different predictions about the causality. So, to experimentally verify fundamental physical theories, we need to be able to experimentally verify the corresponding space-time causality. In other words, we must be able to experimentally check, for every two spacetime events $a$ and $b$, whether the event $a$ can causally influence the event $b$.

Need for a theory-free verification of space-time causality. Since the space-time causality is fundamental, more fundamental than specific partial differential equations that describe the physical fields and/or their relation with space-time, it is desirable to be able to experimentally check this causality in a theory-free way, without invoking other fields and corresponding differential equations.

In this paper, we describe a possible way of such theory-free experimental validation of space-time causality.

Comment. Some of the ideas described in this paper first appeared in [4].

\section{Defining Causality: Challenge}

Intuitive meaning of space-time casuality. Intuitively, the fact that a space-time event $e$ can causally influence an event $e^{\prime}$ means that:

- what we do in the vicinity of $e$

- changes what we observe at $e^{\prime}$.

How to transform this meaning into a definition: a hypothetical idea. The above intuitive meaning of space-time causality can easily lead to a observational definition if we had two (or more) copies of the Universe. In this case, to check 
that $e$ can causally influence $e^{\prime}$, we could do the following (see, e.g., [5]):

- in one copy of the Universe, we perform some action at $e$, and

- we do not perform this action in the second copy of the Universe.

If the resulting states at $e^{\prime}$ are different in the two copies of the Universe, this would be an indication of causal relation between $e$ and $e^{\prime}$.

Comment. This interpretation of causality is known as a counterfactual interpretation; see, e.g., [6]. This name comes from the usual interpretation of counterfactual statements, i.e., statements of the type "If we were born in Sahara, we would have been better adjusted for the warm climate." These statements are called counterfactual because the premise (we are born in Sahara) contradicts to the facts. The usual interpretation of such statements is to consider not just our world, but also the whole set of possible worlds. To check whether a counterfactual statement is true we select, among all possible worlds in which the premise is satisfied, the one which is the closest to our own world. The statement is considered true if the conclusion holds in this selected world.

Similarly, in the counterfactual interpretation of causality, instead of considering only one world, we consider all possible worlds. We then say that $e$ casually influences $e^{\prime}$ if in every world in which $e$ occurs, this occurrence affects $e^{\prime}$. For example, we want to check whether a rain dance $(e)$ causes rain $\left(e^{\prime}\right)$. In our world, we observe a rain dance, and we observe rain, but we cannot tell whether the rain was caused by the rain dance or not. Intuitively, the way to check is to see if rain dances leads to rain. So, in one possible world, we perform a rain dance, in another possible world, we do not perform it. If, as a result, we see rain in the first world but not in the second one, this is good indication that the rain dance indeed causes rain.

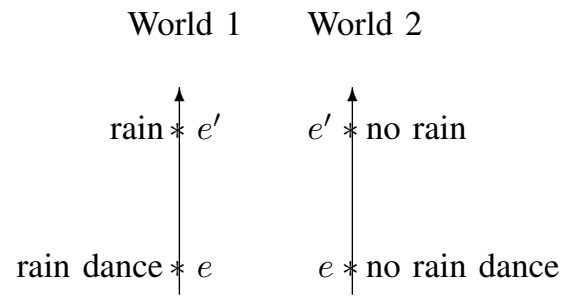

Can we make this idea practical? In reality, we only observe one Universe, in which we either perform the action or we do not.

At first glance, it may seem that in this case, there is no meaningful way to provide an operational definition of spacetime causality.

Our idea. In this paper, we show that a meaningful operational definition of space-time causality is possible if we use the notions of algorithmic randomness and Kolmogorov complexity. Before we explain our idea, let us briefly recall the corresponding notions.

\section{Algorithmic RAndomness And Kolmogorov COMPLEXITY: A BRIEF REMINDER}

Motivation for algorithmic randomness and Kolmogorov complexity. Intuitively, we know that some sequences of $0 \mathrm{~s}$ and $1 \mathrm{~s}$ (or, equivalently, heads and tails) are random, while other sequences are not random. For example, intuitively, if we flip a fair coin 4 times, we can get a sequence 0000 of all heads. However, if we flip a coin 1000 times and still get get all heads, common sense tells us that this coin is not fair. Similarly, common sense tells us that, if we repeatedly flip a fair coin, we cannot expect a periodic sequence $0101 \ldots 01$ (repeated 500 times).

In the traditional probability theory, it is not possible to formally describe this intuitive difference between random and non-random sequences. To provide such a formalization, in the 1960s, A. N. Kolmogorov, one of the world leading specialists in probability theory and mathematical statistics, proposed the following idea (for details, see, e.g., [2]).

Main idea behind algorithmic randomness and Kolmogorov complexity. According to Kolmogorov, the intuitive reason why a sequence consisting of all $0 \mathrm{~s}$ is not random is that it has a simple structure; in other words, this sequence can be generated by a very simple for-loop program. Similarly, a sequence consisting of a sequence 01 repeated 500 times is simple to generate: just make a for-loop. In contrast, a truly random sequence should not have a simple structure - that would enables us to generate it by using some short program: in effect, the shortest way to print this truly random sequence is to actually print it bit-by-bit: print $f(01 \ldots)$.

This idea leads to the following way of detecting whether a given finite sequence $x$ of $0 \mathrm{~s}$ and $1 \mathrm{~s}$ is random or not:

- if this sequence $x$ can be generated by a short program - i.e., a program whose length is much smaller than the length len $(x)$ of this sequence - then $x$ is clearly not random;

- vice versa, if the only way to generate a sequence $x$ is to use a program whose length is at least len $(x)$, then this sequence $x$ is truly random.

To make this distinction, we need to know, for each sequence $x$, the shortest length of a program that generates $x$. This shortest length is known as the Kolmogorov complexity of the string $x$ and denoted by $K(x)$. In terms of Kolmogorov complexity:

- if $K(x) \ll \operatorname{len}(x)$, then $x$ is not random;

- if $K(x) \approx \operatorname{len}(x)$, then $x$ is random.

For example, we can select a small integer $C>0$ (e.g., $C=$ 10 ), and define a sequence $x$ to be random if

$$
K(x) \geq \operatorname{len}(x)-C .
$$

Definition 1. Let a programming language be fixed. By a Kolmogorov complexity $K(x)$ of a finite binary string $x$, we mean the shortest length of a program that generates $x$ :

$$
K(x) \stackrel{\text { def }}{=} \min \{\operatorname{len}(p): p \text { generates } x\} .
$$


Definition 2. Let an integer $C>0$ be fixed. We say that a string $x$ is random if $K(x) \geq \operatorname{len}(x)-C$.

Historical comment. Simultaneously with Kolmogorov, similar ideas were proposed by R. Solomonoff and G. Chaitin [2].

Comment. The length of a program depends on the programming language. However, as Kolmogorov has shown, the definitions $K_{1}(x)$ and $K_{2}(x)$ of Kolmogorov complexity based on two different languages differ by a constant: $\left|K_{1}(x)-K_{2}(x)\right| \leq C_{12}$ for all $x$. Thus, in effect, different programming languages lead to the same definition of a random sequence.

The corresponding notion of independence. In probability theory, in addition to analyzing what is random and what is not, it is also important to decide when the two events are independent and when they are not. Once we have two finite binary sequences $x$ and $y$, the idea that $y$ is independent on $x$ can be described in a similar way:

- if $y$ is independent on $x$, then knowing $x$ does not help us generate $y$;

- in contrast, if $y$ depends on $x$, then knowing $x$ can help us compute $y$.

For example, if we know the locations and velocities $x$ of a mechanical system at some moment of time $t$, we can use this information to easily compute the locations and velocities $y$ at the next moment of time $t+\Delta t$. In contrast, an irrelevant information $x$ (e.g., locations and velocities of particles on another planet) does not help in computing $y$.

To formalize this intuition, we should consider programs that use $x$ as an input to generate $y$.

Definition 3. Let a programming language be fixed. By a relative Kolmogorov complexity $K(y \mid x)$ of a finite binary string $y$ in relation to a binary string $x$, we mean the shortest length of a program that, when using $x$ as an input, generates $y$ :

$$
K(y \mid x) \stackrel{\text { def }}{=} \min \{\operatorname{len}(p): p(x) \text { generates } y\} .
$$

Comment. Intuitively, if using $x$ helps to compute $y$, i.e., if $K(y \mid x) \ll K(y)$, this means that $y$ depends on $x$. Vice versa, if using $x$ does not help to compute $y$, i.e., if $K(y \mid x) \approx K(y)$, this means that $x$ and $y$ are independent. We can describe this in a way similar to the above definition of randomness:

Definition 4. Let an integer $C>0$ be fixed.

- We say that a string $y$ is independent of the string $x$ if

$$
K(y \mid x) \geq K(y)-C .
$$

- We say that a string $y$ is dependent on the string $x$ if

$$
K(y \mid x)<K(y)-C .
$$

IV. How to Define Space-Time Causality: Analysis of THE PRoblem AND THE RESUlting DeFinition

First seeming reasonable idea. At first glance, the above notion of dependence can already lead to a natural definition of space-time causality:

- First, we perform some measurements and observations in the vicinity of the event $e$. Since most nowadays measuring instruments are computer-connected, each such measurement produces a computer-readable output. In the computer, everything is represented as a sequence of $0 \mathrm{~s}$ and $1 \mathrm{~s}$, so the results of all the measurements and observations will also be represented as a sequence $x$ of 0 s and $1 \mathrm{~s}$.

- We also perform measurements and observations in the vicinity of the event $e^{\prime}$, and also produce a sequence $x^{\prime}$ of $0 \mathrm{~s}$ and $1 \mathrm{~s}$.

- If $x^{\prime}$ depends on $x$, i.e., if $K\left(x^{\prime} \mid x\right) \ll K\left(x^{\prime}\right)$, then we claim that $e$ can casually influence $e^{\prime}$.

Unfortunately, this idea does not always work. Yes, if $e$ can casually influence $e^{\prime}$, then we indeed expect that knowing what happened at $e$ can help us predict what is happening at $e^{\prime}$. However, the inverse is not necessarily true: we may have identical observations $x=x^{\prime}$ at events $e$ and $e^{\prime}$ simply because they are both caused by the same event $e^{\prime \prime}$ from the joint past of events $e$ and $e^{\prime}$.

For example, if two people at different locations are watching the same movie, then their observations are identical, but not because they causally influence each other, but because they are both influenced by a past event $e^{\prime \prime}$ (of making this movie).

How to transform the above idea into a working definition. According to modern physics, the Universe is quantum in nature. For many measurements involving microscopic objects, we cannot predict the exact measurement results, we can only predict probabilities of different outcomes. The actual observations are truly random.

Moreover, for each space-time event $e$, we can always set up such random-producing experiments in the small vicinity of $e$, and generate a random sequence $r_{e}$. For example, we can locally set up a Stern-Gerlach experiment (see, e.g., [1]), a quantum experiment that generates a truly random sequence.

This random sequence can affect future results, so if we know this random sequence, it may help us predict future observations. So, if $e$ can causally influence $e^{\prime}$, then for some observations $x^{\prime}$ performed in the small vicinity of $e^{\prime}$, we have $K\left(x^{\prime} \mid r_{e}\right) \ll K\left(x^{\prime}\right)$.

However, it is clear that this sequence cannot affect the measurement results which are in the past (or, more generally, not in the future) of the event $e$. So, if $e$ cannot causally influence $e^{\prime}$, then observations $x^{\prime}$ made in the vicinity of $e^{\prime}$ are independent on $r_{e}: K\left(x^{\prime} \mid r_{e}\right) \approx K\left(x^{\prime}\right)$. So, we arrive at the following semi-formal definition:

Definition 5. For each space-time event e, let $r_{e}$ denote a random sequence that is generated by an experiment performed 
in the small vicinity of $e$. We say that the event e can causally influence the event $e^{\prime}$ if for some observations $x^{\prime}$ performed in the small vicinity of $e^{\prime}$, we have

$$
K\left(x^{\prime} \mid r_{e}\right) \ll K\left(x^{\prime}\right) .
$$

Historical comment. Our definition follows the ideas of casuality as mark transmission [6], [7], with the random sequence as a mark.

Discussion. We have argued that if $e$ does not causally influence $e^{\prime}$, then, no matter what we measure in the vicinity of the event $e^{\prime}$, we get $K\left(x^{\prime} \mid r_{e}\right) \approx K\left(x^{\prime}\right)$; so, in these cases, the above definition is in accordance with the physical intuition.

On the other hand, if $e$ can causally influence $e^{\prime}$, this means that we can send a signal from $e$ to $e^{\prime}$, and as this signal, we send all the bits forming the random sequence $r_{e}$. The signal $x^{\prime}$ received in the vicinity of $e^{\prime}$ will thus be identical to $r_{e}$, so generating $x^{\prime}$ based on $r_{e}$ does not require any computations at all: $K\left(x^{\prime} \mid r_{e}\right)=0$. Since the sequence $x^{\prime}=r_{e}$ is random, we have

$$
K\left(x^{\prime}\right) \geq \operatorname{len}\left(x^{\prime}\right)-C .
$$

For a sufficiently long random sequence $r_{e}=x^{\prime}$, namely for a sequence for which len $\left(x^{\prime}\right)>2 C$, we have

$$
K\left(x^{\prime}\right) \geq \operatorname{len}\left(x^{\prime}\right)-C>2 C-C=C,
$$

so

$$
0=K\left(x^{\prime} \mid r_{e}\right)<K\left(x^{\prime}\right)-C
$$

and thus,

$$
K\left(x^{\prime} \mid r_{e}\right) \ll K\left(x^{\prime}\right) .
$$

So, in these cases, the above definition is also in accordance with the physical intuition.

\section{A Somewhat Unexpected Corollary of Our Definition: SpaCe-Time Causality is a MatTer of DEGREE}

Randomness is a matter of degree. According to the above definition, a sequence $x$ is random if $K(x) \geq \operatorname{len}(x)-C$ for some small integer $C$. Smallness is not an absolute property, it is a matter of degree.

For a given sequence $x$, its degree of randomness $d(x)$ can be described by the smallest integer $C$ for which $K(x) \geq$ len $(x)-C$. One can check that this smallest integer is equal to the difference $d(x)=\operatorname{len}(x)-K(x)$. For random sequences, $d(x)$ is small, for sequences which are not random, this degree is large. The smaller the difference $d(x)$, the more random is the sequence $x$.

So:

- if for some sequence $x$, we have $K(x) \leq \operatorname{len}(x)-C$ for a small integer $C$, while

- for another sequence $x^{\prime}$ of the same length, we only have the inequality $K\left(x^{\prime}\right) \geq \operatorname{len}\left(x^{\prime}\right)-C^{\prime}$ for a larger integer $C^{\prime}>C$, then it is reasonable to conclude that the sequence $x$ is "more random" than the sequence $x^{\prime}$.

For example, if we start with a sequence $x$ obtained by a truly random physical process (e.g., flipping a coin, or, better yet, a truly random quantum experiment) and start replacing the first few bits with 0 s, then we get new sequences which are, intuitively, less and less random - until we replace so many bits by 0 s that the sequence stops being random.

Observation: space-time causality is a matter of degree. Our definition of causality uses the notion of randomness: namely, we say that there is a causal relation between $e$ and $e^{\prime}$ if for some random sequence $r_{e}$ generated in the vicinity of the event $e$ and for measurement results $x^{\prime}$ produced in the vicinity of $e^{\prime}$, we have $K\left(x^{\prime} \mid r_{e}\right)<K\left(x^{\prime}\right)-C$ for some large integer $C$.

The larger the integer $C$, the more confident we are that an event $e$ can causally influence $e^{\prime}$. It is therefore reasonable, for each pair of events $e$ and $e^{\prime}$, to define a degree of causality $c$ as the largest integer $C$ for which $K\left(x^{\prime} \mid r_{e}\right)<K\left(x^{\prime}\right)-C$. One can check that this largest integer is equal to the difference $c=K\left(x^{\prime}\right)-K\left(x^{\prime} \mid r_{e}\right)-1$. The largest this difference $c$, the more confident we are that $e$ can influence $e^{\prime}$. Thus, this difference can serve as degree with which $e$ can influence $e^{\prime}$.

In other words, just like randomness turns out to be a matter of degree, causality is also a matter of degree.

Comment. While this conclusion may be unexpected from the viewpoint of traditional physics, it is in good accordance with the general idea of fuzzy logic, that everything - including causality - is a matter of degree; see, e.g., [8].

Corresponding open problems. It is desirable to explore possible physical meaning of such "degrees of causality": instead of describing the space-time causality, we now have a function $d\left(e, e^{\prime}\right)$ that, for each pair of events for which $e$ causally precedes $e^{\prime}$, describes to what extent $e$ can influence $e^{\prime}$. Maybe this function $d\left(e, e^{\prime}\right)$ is related to relativistic metric - the amount of proper time between $e$ and $e^{\prime}$ ?

Another open problem is related to the fact that he above definition works for localized objects, objects which are located in a small vicinity of one spatial location.

In quantum physics, not all objects are localize in spacetime. We can have situations when the states of two spatially separated particles are entangled. It is desirable to extend our definition to such objects as well.

\section{Conclusions}

In this paper, we propose a new operationalist definition of causality between space-time events. Namely, to check whether an event $e$ can casually influence an event $e^{\prime}$, we:

- generate a truly random sequence $r_{e}$ in the small vicinity of the event $e$, and

- perform observations in the small vicinity of the event $e^{\prime}$.

If some observation results $x^{\prime}$ (obtained near $e^{\prime}$ ) depend on the sequence $r_{e}$ (in the precise sense of dependence described in the paper), then we claim that $e$ can casually influence $e^{\prime}$. 
On the other hand, if all observation results $x^{\prime}$ are independent on $r_{e}$, then we claim that $e$ cannot casually influence $e^{\prime}$.

This new definition naturally leads to a conclusion that space-time causality is a matter of degree, a conclusion that is worth physical analysis.

\section{ACKNOWLEDGMENTS}

This work was supported in part by the National Science Foundation grants HRD-0734825 (Cyber-ShARE Center of Excellence) and DUE-0926721, by Grant 1 T36 GM07800001 from the National Institutes of Health, and by a grant on F-transforms from the Office of Naval Research.

The authors are thankful to John Symons for valuable discussions, and to the anonymous referees for useful suggestions.

\section{REFERENCES}

[1] R. Feynman, R. Leighton, and M. Sands, The Feynman Lectures on Physics, Addison Wesley, Boston, Massachusetts, 2005.

[2] M. Li and P. Vitanyi, An Introduction to Kolmogorov Complexity and Its Applications, Springer, Berlin, Heidelberg, New York, 2008.

[3] C. W. Misner, K. S. Thorne, and J. A. Wheeler, Gravitation, W. H. Freeman, New York, 1973.

[4] A. Ortiz, V. Kreinovich, and J. Symons, How to Define Causality Relation Between Space-Time Events?, working paper.

[5] J. Pearl, Causality: Models, Reasoning, and Inference, Cambridge University Press, Cambridge, UK, 2000

[6] S. Psillos, Causality and Explanation, McGill-Queen's University Press, Montreal, Kingston, and Ithaca, 2002.

[7] W. Salmon, Causality and Explanation, Oxford University Press, Oxford, UK, 1997.

[8] L. A. Zadeh, "Causality is Undefinable: Toward a Theory of Hierarchical Definability", Proceedings of the IEEE International Conference on Fuzzy Systems FUZZ-IEEE'2001, pp. 67-68. 